\title{
Suzuki-type fixed point results in metric type spaces
}

Nawab Hussain ${ }^{*}$, Dragan Đorić 2 , Zoran Kadelburg ${ }^{3}$ and Stojan Radenović ${ }^{4}$

"Correspondence:

nhusain@kau.edu.sa

${ }^{1}$ Department of Mathematics, King Abdulaziz University, P.O. Box 80203,

Jeddah, 21589, Saudi Arabia

Full list of author information is

available at the end of the article

\begin{abstract}
Suzuki's fixed point results from (Suzuki, Proc. Am. Math. Soc. 136:1861-1869, 2008) and (Suzuki, Nonlinear Anal. 71:5313-5317, 2009) are extended to the case of metric type spaces and cone metric type spaces. Examples are given to distinguish our results from the known ones.
\end{abstract}

MSC: $47 \mathrm{H} 10 ; 54 \mathrm{H} 25$

Keywords: metric type space; cone metric space; normal cone; fixed point

\section{Introduction and preliminaries}

In 2008 Suzuki proved the following refinement of Banach's fixed point principle.

Theorem 1 ([1, Theorem 2]) Let $(X, d)$ be a complete metric space. Let $T: X \rightarrow X$ be a selfmap and $\theta_{1}:[0,1) \rightarrow(1 / 2,1]$ be defined by

$$
\theta_{1}(r)= \begin{cases}1, & 0 \leq r \leq \frac{\sqrt{5}-1}{2} \\ \frac{1-r}{r^{2}}, & \frac{\sqrt{5}-1}{2} \leq r \leq \frac{1}{\sqrt{2}} \\ \frac{1}{1+r}, & \frac{1}{\sqrt{2}} \leq r<1\end{cases}
$$

If there exists $r \in[0,1)$ such that for each $x, y \in X$,

$$
\theta_{1}(r) d(x, T x) \leq d(x, y) \quad \Longrightarrow \quad d(T x, T y) \leq r d(x, y),
$$

then $T$ has a unique fixed point $z \in X$ and for each $x \in X$, the sequence $\left\{T^{n} x\right\}$ converges to $z$.

There were various extensions of Suzuki's result, such as Kikkawa-Suzuki's version of Kannan's theorem [2] and Popescu's version of Ćirić's theorem [3].

Suzuki proved also the following version of Edelstein's fixed point theorem.

Theorem 2 ([4, Theorem 3]) Let $(X, d)$ be a compact metric space. Let $T: X \rightarrow X$ be a selfmap, satisfying for all $x, y \in X, x \neq y$ the condition

$$
\frac{1}{2} d(x, T x)<d(x, y) \quad \Longrightarrow \quad d(T x, T y)<d(x, y) .
$$

Then $T$ has a unique fixed point in $X$.

\section{Springer}

(c) 2012 Hussain et al: licensee Springer. This is an Open Access article distributed under the terms of the Creative Commons Attribution License (http://creativecommons.org/licenses/by/2.0), which permits unrestricted use, distribution, and reproduction in any medium, provided the original work is properly cited. 
This theorem was generalized in [5].

Let $E$ be a real Banach space with the zero vector $\theta$. A subset $P$ of $E$ is called a cone if: (a) $P$ is closed, non-empty and $P \neq\{\theta\}$; (b) $a, b \in \mathbb{R}, a, b \geq 0, x, y \in P$ imply that $a x+b y \in P$; (c) $P \cap(-P)=\{\theta\}$. Given a cone $P$, we define the partial ordering $\preceq$ with respect to $P$ by $x \preceq y$ if and only if $y-x \in P$. We shall write $x \ll y$ for $y-x \in \operatorname{int} P$, where int $P$ stands for the interior of $P$ and use $x \prec y$ for $x \preceq y$ and $x \neq y$. If int $P \neq \emptyset$, then $P$ is called a solid cone. It is said to be normal if there is a number $K>0$ such that for all $x, y \in E, \theta \preceq x \preceq y$ implies $\|x\| \leq K\|y\|$. Such a minimal constant $K$ is called the normal constant of $P$.

Huang and Zhang re-introduced cone metric spaces in [6] (this notion was known under various names since the mid of the 20th century, see a survey in [7]), replacing the set of real numbers by an ordered Banach space as the codomain for a metric. Cone metric spaces over normal cones inspired another generalization of metric spaces that were called metric type spaces by Khamsi [8] (see also [9-12]; note that, in fact, spaces of this kind were used earlier under the name of $b$-spaces by Czerwik [13]). Cvetković et al. [14] and Shah et al. [15] extended Khamsi's definition and defined cone metric type spaces as follows:

Definition $1([14,15])$ Let $X$ be a nonempty set, $E$ a Banach space with the solid cone $P$ and let $K \geq 1$ be a real number. If the function $D: X \times X \rightarrow P$ satisfies the following properties:

(a) $D(x, y)=0$ if and only if $x=y$;

(b) $D(x, y)=D(y, x)$ for all $x, y \in X$;

(c) $D(x, z) \preceq K(D(x, y)+D(y, z))$ for all $x, y, z \in X$,

then $D$ is called a cone metric type function and $(X, D, K)$ is called a cone metric type space (CMTS).

In particular, when $E=\mathbb{R}$ and $P=[0,+\infty)$, CMTS $(X, D, K)$ reduces to a metric type space (MTS) of $[8,9,12]$.

Of course, for $K=1$ we get the cone metric space (CMS) of [6], resp. the usual metric space.

Example 1 ([14]) Let $B=\left\{e_{1}, \ldots, e_{n}\right\}$ be an orthonormal basis of $\mathbb{R}^{n}$ with inner product $\langle\cdot, \cdot\rangle$ and let $p>0$. Define

$$
X_{p}=\left\{\left.[f]\left|f:[0,1] \rightarrow \mathbb{R}^{n}, \int_{0}^{1}\right|\left\langle f(t), e_{k}\right\rangle\right|^{p} d t<+\infty, k=1, \ldots, n\right\},
$$

where $[f]$ is the class of functions being equal to the function $f$ a.e. Further, let

$$
P_{B}=\left\{y \in \mathbb{R}^{n} \mid\left\langle y, e_{k}\right\rangle \geq 0, k=1,2, \ldots, n\right\},
$$

and let $D: X_{p} \times X_{p} \rightarrow P_{B}$ be defined by

$$
D_{p}(f, g)=\sum_{k=1}^{n} e_{k} \int_{0}^{1}\left|\left\langle f(t)-g(t), e_{k}\right\rangle\right|^{p} d t, \quad f, g \in X_{p}
$$

It was shown in [14] that $P_{B}$ is a solid cone in $\mathbb{R}^{n}$ and that $\left(X_{p}, D_{p}, 2^{p-1}\right)$ is a CMTS. In particular, for $n=1$ we get an MTS and for $p=1$ a CMS. 
Example $2([8,10])$ Let $(X, d)$ be any CMS over a normal cone with normal constant $K \geq 1$. Then $(X, D, K)$ is an MTS, where $D(x, y)=\|d(x, y)\|$. In this case the spaces $(X, d)$ and $(X, D, K)$ have the same topologies (see [10, Theorem 2.7]).

If $\left(X, D_{1}, K\right)$ is a CMTS over a normal cone with a normal constant $k \geq 1$, then $(X, D, K k)$ is an MTS, where $D(x, y)=\left\|D_{1}(x, y)\right\|$. Similarly as above, the spaces $\left(X, D_{1}, K\right)$ and $(X, D, K k)$ have the same topologies.

Notions such as convergent and Cauchy sequences, as well as completeness, are introduced in (cone) metric type spaces in the standard way. The following obviously holds in an arbitrary (cone) metric type space:

$$
x_{n} \rightarrow x \quad \text { and } y_{n} \rightarrow x \quad \Longrightarrow \quad D\left(x_{n}, y_{n}\right) \rightarrow 0 \text {. }
$$

We will sometimes need the continuity of metric-type function $D$ in one variable:

$$
x_{n} \rightarrow x \quad \Longrightarrow \quad D\left(x_{n}, y\right) \rightarrow D(x, y)
$$

or in two variables:

$$
x_{n} \rightarrow x \quad \text { and } \quad y_{n} \rightarrow y \quad \Longrightarrow \quad D\left(x_{n}, y_{n}\right) \rightarrow D(x, y) .
$$

The last property always holds in the case of an MTS $(X, D, K)$ generated by a CMS $(X, d)$ over a normal cone, see Example 2, but not in general, as the following example shows.

Example 3 Let $X=\mathbb{N} \cup\{\infty\}$ and let $D: X \times X \rightarrow \mathbb{R}$ be defined by

$$
D(m, n)= \begin{cases}0, & \text { if } m=n, \\ \left|\frac{1}{m}-\frac{1}{n}\right|, & \text { if } m \text { and } n \text { are even or } m n=\infty \\ 5, & \text { if } m \text { and } n \text { are odd and } m \neq n \\ 2, & \text { otherwise. }\end{cases}
$$

Then it is easy to see that for all $m, n, p \in X$, we have

$$
D(m, p) \leq 3(D(m, n)+D(n, p))
$$

Thus, $(X, D, 3)$ is a metric-type space. Let $x_{n}=2 n$ for each $n \in \mathbb{N}$. Then

$$
D(2 n, \infty)=\frac{1}{2 n} \rightarrow 0 \quad \text { as } n \rightarrow \infty
$$

that is, $x_{n} \rightarrow \infty$, but $D\left(x_{n}, 1\right)=2 \nrightarrow \rightarrow D(\infty, 1)$ as $n \rightarrow \infty$.

Recall that a selfmap $T: X \rightarrow X$ is said to have the property $(P)[16]$ if $F(T)=F\left(T^{n}\right)$ for each $n \in \mathbb{N}$, where $F(T)$ is the set of fixed points of $T$.

In this paper, we extend Suzuki's Theorems 1 and 2, as well as Popescu's results from [3] to the case of metric type spaces and cone metric type spaces. Examples are given to distinguish our results from the known ones. 


\section{Results}

\subsection{Results in metric type spaces}

Theorem 3 Let $(X, D, K)$ be a complete MTS where $D$ is continuous in each variable. Let $T: X \rightarrow X$ be a selfmap and $\theta=\theta_{K}:[0,1) \rightarrow(1 /(K+1), 1]$ be defined by

$$
\theta(r)=\theta_{K}(r)= \begin{cases}1, & 0 \leq r \leq \frac{\sqrt{5}-1}{2} \\ \frac{1-r}{r^{2}}, & \frac{\sqrt{5}-1}{2} \leq r \leq b_{K} \\ \frac{1}{K+r}, & b_{K} \leq r<1\end{cases}
$$

where $b_{K}=\frac{1-K+\sqrt{1+6 K+K^{2}}}{4}$ is the positive solution of $\frac{1-r}{r^{2}}=\frac{1}{K+r}$. If there exists $r \in[0,1)$ such that for each $x, y \in X$,

$$
\theta(r) D(x, T x) \leq D(x, y) \quad \Longrightarrow \quad D(T x, T y) \leq \frac{r}{K} M(x, y),
$$

where

$$
M(x, y)=\max \left\{D(x, y), D(x, T x), D(y, T y), \frac{1}{2 K}(D(x, T y)+D(y, T x))\right\},
$$

then $T$ has a unique fixed point $z \in X$ and for each $x \in X$, the sequence $\left\{T^{n} x\right\}$ converges to $z$. Moreover, $T$ has the property (P).

Note that for $K=1$, Theorem 3 reduces to a special case of Theorem 2.1 by Popescu [3].

Proof First note that $\theta(r) \leq 1$ implies that $\theta(r) D(x, T x) \leq D(x, T x)$ and it follows by (2.2) that

$$
\begin{aligned}
D\left(T x, T^{2} x\right) & \leq \frac{r}{K} \max \left\{D(x, T x), D\left(T x, T^{2} x\right), \frac{1}{2 K} D\left(x, T^{2} x\right)\right\} \\
& \leq \frac{r}{K} \max \left\{D(x, T x), D\left(T x, T^{2} x\right), \frac{1}{2}\left(D(x, T x)+D\left(T x, T^{2} x\right)\right)\right\} \\
& =\frac{r}{K} \max \left\{D(x, T x), D\left(T x, T^{2} x\right)\right\},
\end{aligned}
$$

wherefrom

$$
D\left(T x, T^{2} x\right) \leq \frac{r}{K} D(x, T x)
$$

for each $x \in X$.

Let $u \in X$ be arbitrary and form the sequence $\left\{u_{n}\right\}$ by $u_{0}=u$ and $u_{n}=T^{n} u$ for $n \in \mathbb{N}$. It follows from (2.3) that

$$
D\left(u_{n}, u_{n+1}\right) \leq \frac{r}{K} D\left(u_{n-1}, u_{n}\right)
$$

and, by induction,

$$
D\left(u_{n}, u_{n+1}\right) \leq \frac{r^{n}}{K^{n}} D(u, T u) .
$$


Using [12, Lemma 3.1] we conclude that $\left\{u_{n}\right\}$ is a Cauchy sequence, tending to some $z$ in the complete space $X$. Obviously, also $T u_{n}=u_{n+1} \rightarrow z, n \rightarrow \infty$.

Let us prove now that

$$
D(T x, z) \leq \frac{r}{K} \max \{D(x, z), D(x, T x)\}
$$

holds for each $x \neq z$. Since $u_{n} \rightarrow z$ and $T u_{n} \rightarrow z$ (and hence $D\left(u_{n}, T u_{n}\right) \rightarrow 0$ ) and, by continuity of $D, D\left(u_{n}, x\right) \rightarrow D(x, z) \neq 0$, it follows that there exists $n_{0} \in \mathbb{N}$ such that

$$
\theta(r) D\left(u_{n}, T u_{n}\right)<D\left(u_{n}, x\right)
$$

holds for each $n \geq n_{0}$. Assumption (2.2) implies that for such $n$

$$
\begin{aligned}
D\left(T u_{n}, T x\right) \leq & \frac{r}{K} \max \left\{D\left(u_{n}, x\right), D\left(u_{n}, u_{n+1}\right), D(x, T x),\right. \\
& \left.\frac{1}{2 K}\left(D\left(u_{n}, T x\right)+D\left(u_{n+1}, x\right)\right)\right\} .
\end{aligned}
$$

Passing to the limit when $n \rightarrow \infty$ (and using continuity of $D$ ), we get that

$$
D(T x, z) \leq \frac{r}{K} \max \left\{D(x, z), D(x, T x), \frac{1}{2 K}(D(z, T x)+D(x, z))\right\} .
$$

It is easy to see that (2.6) follows from the previous relation.

Putting $x=T^{n-1} z$ in (2.3), we get that

$$
D\left(T^{n} z, T^{n+1} z\right) \leq \frac{r}{K} D\left(T^{n-1} z, T^{n} z\right)
$$

holds for each $n \in \mathbb{N}$ (where $T^{0} z=z$ ). It follows by induction that

$$
D\left(T^{n} z, T^{n+1} z\right) \leq \frac{r^{n}}{K^{n}} D(z, T z) .
$$

We will prove now that

$$
D\left(T^{n} z, z\right) \leq D(T z, z)
$$

for each $n \in \mathbb{N}$. For $n=1$ this relation is obvious. Suppose that it holds for some $n \in \mathbb{N}$. If $T^{n} z=z$, then $T^{n+1} z=T z$ and $D\left(T^{n+1} z, z\right)=D(T z, z) \leq D(T z, z)$. If $T^{n} z \neq z$, then we can apply (2.6) to obtain that

$$
D\left(T^{n+1} z, z\right) \leq \frac{r}{K} \max \left\{D\left(T^{n} z, z\right), D\left(T^{n} z, T^{n+1} z\right)\right\} .
$$

Using (2.8) and the induction hypothesis, we get that

$$
D\left(T^{n+1} z, z\right) \leq \frac{r}{K} \max \left\{D(T z, z), \frac{r^{n}}{K^{n}} D(T z, z)\right\}=\frac{r}{K} D(T z, z) \leq d(T z, z),
$$

and (2.9) is proved by induction. 
In order to prove that $T z=z$, we suppose that $T z \neq z$ and consider the two possible cases. Case I. $0 \leq r<b_{K}$ (and hence $\theta(r) \leq \frac{1-r}{r^{2}}$ ). We will prove first that

$$
D\left(T^{n} z, T z\right) \leq \frac{r}{K} D(T z, z)
$$

for $n \in \mathbb{N}$. For $n=1$ this is obvious and for $n=2$ it follows from (2.8). Suppose that (2.10) holds for some $n \geq 2$. Then

$$
D(z, T z) \leq K\left(D\left(z, T^{n} z\right)+D\left(T^{n} z, T z\right)\right) \leq K\left(D\left(z, T^{n} z\right)+\frac{r}{K} D(T z, z)\right),
$$

wherefrom $D(z, T z) \leq \frac{K}{1-r} D\left(z, T^{n} z\right)$. It follows (using (2.8)) that

$$
\begin{aligned}
\theta(r) D\left(T^{n} z, T^{n+1} z\right) & \leq \frac{1-r}{r^{2}} D\left(T^{n} z, T^{n+1} z\right) \leq \frac{1-r}{r^{n}} D\left(T^{n} z, T^{n+1} z\right) \\
& \leq \frac{1-r}{K^{n}} D(z, T z) \leq \frac{1}{K^{n-1}} D\left(z, T^{n} z\right) \leq D\left(T^{n} z, z\right) .
\end{aligned}
$$

Assumption (2.2) implies that

$$
\begin{aligned}
D\left(T^{n+1} z, T z\right) \leq & \frac{r}{K} \max \left\{D\left(T^{n} z, z\right), D\left(T^{n} z, T^{n+1} z\right), D(z, T z),\right. \\
& \left.\frac{1}{2 K}\left(D\left(z, T^{n+1} z\right)+D\left(T z, T^{n} z\right)\right)\right\} .
\end{aligned}
$$

It is easy to see (using (2.8), (2.9) and the inductive hypothesis) that the last maximum is equal to $D(z, T z)$, i.e., $D\left(T^{n+1} z, T z\right) \leq \frac{r}{K} D(z, T z)$ and relation (2.10) is proved by induction.

Now $T z \neq z$ and (2.10) implies that $T^{n} z \neq z$ for each $n \in \mathbb{N}$. Hence, (2.6) and (2.8) imply that

$$
\begin{aligned}
D\left(T^{n+1} z, z\right) & \leq \frac{r}{K} \max \left\{D\left(T^{n} z, z\right), D\left(T^{n} z, T^{n+1} z\right)\right\} \\
& \leq \frac{r}{K} \max \left\{D\left(T^{n} z, z\right), \frac{r^{n}}{K^{n}} D(z, T z)\right\} .
\end{aligned}
$$

Since $D(T z, z) \leq K\left(D\left(T z, T^{n} z\right)+D\left(T^{n} z, z\right)\right)$, it follows from (2.10) that

$$
D\left(T^{n} z, z\right) \geq \frac{1}{K} D(T z, z)-D\left(T z, T^{n} z\right) \geq \frac{1-r}{K} D(T z, z) .
$$

There exists $n_{1} \in \mathbb{N}$ such that $1-r \geq r^{n}$ for $n \geq n_{1}$ and $0 \leq r<b_{K}$. For such $n$, we have that

$$
D\left(T^{n} z, z\right) \geq \frac{r^{n}}{K} D(T z, z) \geq \frac{r^{n}}{K^{n}} D(T z, z)
$$

It follows from (2.11) that

$$
D\left(T^{n+1} z, z\right) \leq \frac{r}{K} D\left(T^{n} z, z\right) \leq \cdots \leq\left(\frac{r}{K}\right)^{n-n_{1}+1} D\left(T^{n_{1}} z, z\right) \rightarrow 0 \quad(n \rightarrow \infty) .
$$

Thus, $T^{n} z \rightarrow z$ and, again from (2.10), we get that $D(T z, z) \leq \frac{r}{K} D(T z, z)$ and $D(T z, z)=0$, a contradiction. 
Case II. $b_{K} \leq r<1$ (and so $\theta(r)=\frac{1}{K+r}$ ). We will prove that there exists a subsequence $\left\{u_{n_{j}}\right\}$ of $\left\{u_{n}\right\}$ such that

$$
\theta(r) D\left(u_{n_{j}}, T u_{n_{j}}\right)=\theta(r) D\left(u_{n_{j}}, u_{n_{j}+1}\right) \leq D\left(u_{n_{j}}, z\right)
$$

holds for each $j \in \mathbb{N}$. From (2.4) we know that $D\left(u_{n}, u_{n+1}\right) \leq \frac{r}{K} D\left(u_{n-1}, u_{n}\right)$ holds for each $n \in \mathbb{N}$. Suppose that

$$
\frac{1}{K+r} D\left(u_{n-1}, u_{n}\right)>D\left(u_{n-1}, z\right) \text { and } \frac{1}{K+r} D\left(u_{n}, u_{n+1}\right)>D\left(u_{n}, z\right)
$$

both hold for some $n \in \mathbb{N}$. Then

$$
\begin{aligned}
D\left(u_{n-1}, u_{n}\right) & \leq K\left(D\left(u_{n-1}, z\right)+D\left(u_{n}, z\right)\right)<\frac{K}{K+r}\left(D\left(u_{n-1}, z\right)+D\left(u_{n}, u_{n+1}\right)\right) \\
& \leq \frac{K}{K+r}\left(D\left(u_{n-1}, u_{n}\right)+\frac{r}{K} D\left(u_{n-1}, u_{n}\right)\right)=D\left(u_{n-1}, u_{n}\right),
\end{aligned}
$$

which is impossible. Hence one of the following holds for each $n$ :

$$
\theta(r) D\left(u_{n-1}, u_{n}\right) \leq D\left(u_{n-1}, z\right) \quad \text { or } \quad \theta(r) D\left(u_{n}, u_{n+1}\right) \leq D\left(u_{n}, z\right) .
$$

In particular,

$$
\theta(r) D\left(u_{2 n-1}, u_{2 n}\right) \leq D\left(u_{2 n-1}, z\right) \quad \text { or } \quad \theta(r) D\left(u_{2 n}, u_{2 n+1}\right) \leq D\left(u_{2 n}, z\right)
$$

holds for each $n \in \mathbb{N}$. In other words, there is a subsequence $\left\{u_{n_{j}}\right\}$ of $\left\{u_{n}\right\}$ such that (2.12) holds for each $j \in \mathbb{N}$. But then assumption (2.2) implies that

$$
\begin{aligned}
D\left(T u_{n_{j}}, T z\right) \leq & \frac{r}{K} \max \left\{D\left(u_{n_{j}}, z\right), D\left(u_{n_{j}}, T u_{n_{j}}\right), D(z, T z),\right. \\
& \left.\frac{1}{2 K}\left(D\left(u_{n_{j}}, T z\right)+D\left(T u_{n_{j}}, z\right)\right)\right\} .
\end{aligned}
$$

Passing to the limit when $j \rightarrow \infty$ we get that $D(z, T z) \leq \frac{r}{K} D(z, T z)$, which is possible only if $T z=z$, a contradiction.

Thus, we have proved that $z$ is a fixed point of $T$. The uniqueness of the fixed point follows easily from (2.6). Indeed, if $y, z$ are two fixed points of $T$, then (2.6) implies that

$$
D(y, z)=D(T y, z) \leq \frac{r}{K} \max \{D(y, z), D(y, T y)\}=\frac{r}{K} D(y, z),
$$

wherefrom $y=z$. The property $(P)$ follows from (2.3) (see [16]).

Suzuki-Banach-type and Suzuki-Kannan-type fixed point results in metric type spaces (versions of [1, Theorem 2] and [2, Theorem 2.2]) are special cases of Theorem 3.

Corollary 1 Let $(X, D, K)$ be a complete MTS where $D$ is continuous in each variable. Let $T: X \rightarrow X$ be a selfmap and $\theta:[0,1) \rightarrow(1 /(K+1), 1]$ be defined by (2.1). If there exists 
$r \in[0,1)$ such that for each $x, y \in X$,

$$
\theta(r) D(x, T x) \leq D(x, y) \quad \Longrightarrow \quad D(T x, T y) \leq \frac{r}{K} D(x, y),
$$

then $T$ has a unique fixed point $z \in X$ and for each $x \in X$, the sequence $\left\{T^{n} x\right\}$ converges to $z$. Moreover, $T$ has the property $(P)$.

Corollary 2 Let $(X, D, K)$ be a complete MTS where $D$ is continuous in each variable. Let $T: X \rightarrow X$ be a selfmap and $\theta:[0,1) \rightarrow(1 /(K+1), 1]$ be defined by (2.1). If there exists $r \in[0,1)$ such that for each $x, y \in X$,

$$
\theta(r) D(x, T x) \leq D(x, y) \quad \Longrightarrow \quad D(T x, T y) \leq \frac{r}{K} \max \{D(x, T x), D(y, T y)\},
$$

then $T$ has a unique fixed point $z \in X$ and for each $x \in X$, the sequence $\left\{T^{n} x\right\}$ converges to $z$. Moreover, $T$ has the property (P).

Corollary 3 Let $(X, D, K)$ be a complete MTS where $D$ is continuous in each variable. Let $T: X \rightarrow X$ be a selfmap and $\theta:[0,1) \rightarrow(1 /(K+1), 1]$ be defined by $(2.1)$. If there exists $r \in[0,1)$ such that for each $x, y \in X$,

$$
\theta(r) D(x, T x) \leq D(x, y) \quad \Longrightarrow \quad D(T x, T y) \leq \frac{r}{2 K}(D(x, T y)+D(y, T x)),
$$

then $T$ has a unique fixed point $z \in X$ and for each $x \in X$, the sequence $\left\{T^{n} x\right\}$ converges to $z$. Moreover, $T$ has the property (P).

Adapting [1, Example 1] we give now an example of a mapping satisfying the conditions of Theorem 3 (and having a unique fixed point) but not satisfying the respective classical (non-Suzuki-type) condition in metric type spaces (see, e.g., [14, Theorem 3.4]).

Example 4 Let $X=\{(0,0),(4,0),(0,4),(4,6),(6,4)\}$, and let $D: X \times X \rightarrow[0,+\infty)$ be given by $D\left(\left(x_{1}, x_{2}\right),\left(y_{1}, y_{2}\right)\right)=\left(x_{1}-y_{1}\right)^{2}+\left(x_{2}-y_{2}\right)^{2}$. Then $(X, D, 2)$ is a metric type space (see Example 1). Let $T: X \rightarrow X$ be given as

$$
T:\left(\begin{array}{lllll}
(0,0) & (4,0) & (0,4) & (4,6) & (6,4) \\
(0,0) & (0,0) & (0,0) & (4,0) & (0,4)
\end{array}\right) .
$$

We will check that condition (2.2) holds true for $r=8 / 9$ and all $x, y \in X$. If $x=y$ or if $x, y \in$ $\{(0,0),(4,0),(0,4)\}$, it is trivially satisfied. Let $x \in\{(0,0),(4,0),(0,4)\}$ and $y \in\{(4,6),(6,4)\}$. Then $D(T x, T y)=16$ and $M(x, y)=52$ for $x=(0,0)$ and $M(x, y)=36$ for $x=(4,0)$ or $x=$ $(0,4)$. Hence, in any case,

$$
D(T x, T y)=16 \leq \frac{8 / 9}{2} \cdot M(x, y) .
$$

Let now $x, y \in\{(4,6),(6,4)\}, x \neq y$. Then $D(x, y)=8$ and $D(x, T x)=36$ and so $\theta(r) D(x, T x)>$ $\frac{1}{3} \cdot 36>8=D(x, y)$, and (2.2) is trivially satisfied. Note that in the classical variant, in this case $D(T x, T y)=32$ and $M(x, y)=36$, so the inequality $D(T x, T y) \leq \frac{r}{2} M(x, y)$ does not hold for any $r<1$. 
The following is a metric-type version of Theorem 2 .

Theorem 4 Let $(X, D, K)$ be a compact MTS, where the function $D$ is continuous. Let $T$ : $X \rightarrow X$ be a selfmap, satisfying for all $x, y \in X, x \neq y$ the condition

$$
\frac{1}{1+K} D(x, T x)<D(x, y) \quad \Longrightarrow \quad D(T x, T y)<\frac{1}{K} D(x, y) .
$$

Then $T$ has a unique fixed point in $X$.

Proof Denote $\beta=\inf \{D(x, T x): x \in X\}$ and choose a sequence $\left\{x_{n}\right\}$ in $X$ such that $D\left(x_{n}, T x_{n}\right) \rightarrow \beta(n \rightarrow \infty)$. Since the space $X$ is (sequentially) compact, we can suppose that there exist $v, w \in X$ such that $x_{n} \rightarrow v$ and $T x_{n} \rightarrow w(n \rightarrow \infty)$. We will prove that $\beta=0$.

Suppose that $\beta>0$ and note that continuity of $D$ implies that $\lim _{n \rightarrow \infty} D\left(x_{n}, w\right)=$ $D(v, w)=\lim _{n \rightarrow \infty} D\left(x_{n}, T x_{n}\right)=\beta$. Choose $n_{0} \in \mathbb{N}$ such that for all $n \geq n_{0}$

$$
\frac{2+K}{2+2 K} \beta<D\left(x_{n}, w\right) \quad \text { and } \quad D\left(x_{n}, T x_{n}\right)<\frac{2+K}{2} \beta
$$

holds true. Then $\frac{1}{1+K} D\left(x_{n}, T x_{n}\right)<D\left(x_{n}, w\right)$ and assumption (2.13) implies that $D\left(T x_{n}, T w\right)<$ $\frac{1}{K} D\left(x_{n}, w\right)$ for $n \geq n_{0}$. Passing to the limit, we obtain that $D(w, T w) \leq \frac{1}{K} \beta$. If $K>1$, the last inequality is impossible by the definition of $\beta$. If $K=1$, it is possible only if $D(w, T w)=\beta$ (recall that we have supposed that $\beta>0$ ). But in this case $\frac{1}{1+K} D(w, T w)<D(w, T w)$ and (2.13) implies that $D\left(T w, T^{2} w\right)<\frac{1}{K} D(w, T w)=\beta$, which is again impossible by the definition of $\beta$. Hence, in all cases we obtain a contradiction and it follows that $\beta=0$ and so $v=w$.

In order to prove that $T$ has a fixed point, suppose that $T z \neq z$ for all $z \in X$. Then, in particular, $0<\frac{1}{1+K} D\left(x_{n}, T x_{n}\right)<D\left(x_{n}, T x_{n}\right)$ and (2.13) implies that

$$
D\left(T x_{n}, T^{2} x_{n}\right)<\frac{1}{K} D\left(x_{n}, T x_{n}\right) .
$$

It follows that

$$
D\left(v, T^{2} x_{n}\right) \leq K\left(D\left(v, T x_{n}\right)+D\left(T x_{n}, T^{2} x_{n}\right)\right)<K D\left(v, T x_{n}\right)+D\left(x_{n}, T x_{n}\right) \rightarrow 0,
$$

when $n \rightarrow \infty$. Hence, $T^{2} x_{n} \rightarrow v(n \rightarrow \infty)$.

Suppose now that

$$
\frac{1}{1+K} D\left(x_{n}, T x_{n}\right) \geq D\left(x_{n}, v\right) \text { and } \frac{1}{1+K} D\left(T x_{n}, T^{2} x_{n}\right) \geq D\left(T x_{n}, v\right)
$$

both hold for some $n \in \mathbb{N}$. Then

$$
\begin{aligned}
D\left(x_{n}, T x_{n}\right) & \leq K\left(D\left(x_{n}, v\right)+D\left(T x_{n}, v\right)\right) \\
& \leq \frac{K}{1+K}\left(D\left(x_{n}, T x_{n}\right)+D\left(T x_{n}, T^{2} x_{n}\right)\right) \\
& <\frac{K}{1+K}\left(D\left(x_{n}, T x_{n}\right)+\frac{1}{K} D\left(x_{n}, T x_{n}\right)\right)=D\left(x_{n}, T x_{n}\right),
\end{aligned}
$$


which is impossible. Thus, for each $n \in \mathbb{N}$, either

$$
\frac{1}{1+K} D\left(x_{n}, T x_{n}\right)<D\left(x_{n}, v\right) \quad \text { or } \quad \frac{1}{1+K} D\left(T x_{n}, T^{2} x_{n}\right)<D\left(T x_{n}, v\right)
$$

holds true. Assumption (2.13) implies that for each $n \in \mathbb{N}$ either

$$
D\left(T x_{n}, T v\right)<\frac{1}{K} D\left(x_{n}, v\right) \quad \text { or } \quad D\left(T^{2} x_{n}, T v\right)<\frac{1}{K} D\left(T x_{n}, v\right)
$$

holds. In other words, there exists a sequence $\left\{n_{j}\right\}$ such that $D\left(T x_{n_{j}}, T v\right)<\frac{1}{K} D\left(x_{n_{j}}, v\right)$ holds for each $j \in \mathbb{N}$, or there exists a sequence $\left\{n_{k}\right\}$ such that $D\left(T^{2} x_{n_{k}}, T v\right)<\frac{1}{K} D\left(x_{n_{k}}, v\right)$ holds for each $k \in \mathbb{N}$. In both cases, passing to the limit, we obtain that $D(v, T v)=0$, i.e., $T v=v$, a contradiction with the assumption that $T$ has no fixed points.

It follows that there exists $z \in X$ such that $T z=z$. Uniqueness follows easily.

\subsection{Results in cone metric type spaces}

In this subsection, we formulate cone-metric-type versions of the results from the previous subsection.

Theorem 5 Let $\left(X, D_{1}, K\right)$ be a complete $C M T S$ with the normal underlying cone $P$, where $D_{1}$ is continuous in each variable. Let $T: X \rightarrow X$ be a selfmap and $\theta=\theta_{K}:[0,1) \rightarrow(1 /(K+$ $1), 1]$ be defined by (2.1). If there exists $r \in[0,1)$ such that for each $x, y \in X$,

$$
\theta(r) D_{1}(x, T x)-D_{1}(x, y) \notin \operatorname{int} P \quad \Longrightarrow \quad D_{1}(T x, T y) \preceq \frac{r}{K} u(x, y),
$$

for some

$$
u(x, y) \in\left\{D_{1}(x, y), D_{1}(x, T x), D_{1}(y, T y), \frac{1}{2 K}\left(D_{1}(x, T y)+D_{1}(y, T x)\right)\right\}
$$

then $T$ has a unique fixed point $z \in X$ and for each $x \in X$, the sequence $\left\{T^{n} x\right\}$ converges to $z$.

Proof Since the cone $P$ is normal, without loss of generality, we can assume that the normal constant of $P$ is $k=1$ and that the given norm in $E$ is monotone, i.e., $\theta \preceq c \ll d \Longrightarrow$ $\|c\|<\|d\|$ (see [17, Lemma 2.1]). Denote $D(x, y)=\left\|D_{1}(x, y)\right\|$. Then $D$ is a (real-valued) metric-type function and the space $(X, D)$ is compact (together with $\left(X, D_{1}\right)$, see $[10$, Theorem 2.7]). Let us prove that the mapping $T$ satisfies for some $r \in[0,1)$ the condition

$$
(\forall x, y \in X) \quad \theta(r) D(x, T x) \leq D(x, y) \quad \Longrightarrow \quad D(T x, T y) \leq \frac{r}{K} M(x, y)
$$

of Theorem 3. Suppose that $\theta(r) D(x, T x) \leq D(x, y)$. Then $\theta(r) D_{1}(x, T x)-D_{1}(x, y) \notin \operatorname{int} P$ (indeed, if, to the contrary, $\theta(r) D_{1}(x, T x)-D_{1}(x, y) \in \operatorname{int} P$, i.e., $\theta(r) D_{1}(x, T x) \gg D_{1}(x, y)$, it would follow that $\theta(r) D(x, T x)>D(x, y)$, a contradiction with the assumption). Assumption (2.14) implies that $D_{1}(T x, T y) \preceq \frac{r}{K} u(x, y)$ for some

$$
u(x, y) \in\left\{D_{1}(x, y), D_{1}(x, T x), D_{1}(y, T y), \frac{1}{2 K}\left(D_{1}(x, T y)+D_{1}(y, T x)\right)\right\} .
$$


Again by the monotonicity of the norm, this means that $D(T x, T y) \leq \frac{r}{K} M(x, y)$, where

$$
M(x, y)=\max \left\{D(x, y), D(x, T x), D(y, T y), \frac{1}{2 K}(D(x, T y)+D(y, T x))\right\} .
$$

Hence, condition (2.15) is satisfied, and the conclusion follows.

In a similar way, the following corollaries and the theorem can be proved.

Corollary 4 Let $\left(X, D_{1}, K\right)$ be a complete CMTS where $D_{1}$ is continuous in each variable. Let $T: X \rightarrow X$ be a selfmap and $\theta:[0,1) \rightarrow(1 /(K+1), 1]$ be defined by (2.1). If there exists $r \in[0,1)$ such that for each $x, y \in X$,

$$
\theta(r) D_{1}(x, T x)-D_{1}(x, y) \notin \operatorname{int} P \quad \Longrightarrow \quad D_{1}(T x, T y) \preceq \frac{r}{K} D_{1}(x, y),
$$

then $T$ has a unique fixed point $z \in X$ and for each $x \in X$, the sequence $\left\{T^{n} x\right\}$ converges to $z$.

Corollary 5 Let $\left(X, D_{1}, K\right)$ be a complete CMTS where $D_{1}$ is continuous in each variable. Let $T: X \rightarrow X$ be a selfmap and $\theta:[0,1) \rightarrow(1 /(K+1), 1]$ be defined by (2.1). If there exists $r \in[0,1)$ such that for each $x, y \in X$,

$$
\theta(r) D_{1}(x, T x)-D_{1}(x, y) \notin \operatorname{int} P \quad \Longrightarrow \quad D_{1}(T x, T y) \preceq \frac{r}{K} u(x, y),
$$

where $u(x, y) \in\left\{D_{1}(x, T x), D_{1}(y, T y)\right\}$, then $T$ has a unique fixed point $z \in X$ and for each $x \in X$, the sequence $\left\{T^{n} x\right\}$ converges to $z$.

Corollary 6 Let $\left(X, D_{1}, K\right)$ be a complete CMTS where $D_{1}$ is continuous in each variable. Let $T: X \rightarrow X$ be a selfmap and $\theta:[0,1) \rightarrow(1 /(K+1), 1]$ be defined by $(2.1)$. If there exists $r \in[0,1)$ such that for each $x, y \in X$,

$$
\theta(r) D_{1}(x, T x)-D_{1}(x, y) \notin \operatorname{int} P \quad \Longrightarrow \quad D_{1}(T x, T y) \preceq \frac{r}{2 K}\left(D_{1}(x, T y)+D_{1}(y, T x)\right),
$$

then $T$ has a unique fixed point $z \in X$ and for each $x \in X$, the sequence $\left\{T^{n} x\right\}$ converges to $z$.

Example 4 can be easily adapted to a CMTS.

Theorem 6 Let $\left(X, D_{1}, K\right)$ be a compact CMTS, where the function $D_{1}$ is continuous. Let $T: X \rightarrow X$ be a selfmap satisfying, for all $x, y \in X, x \neq y$ the condition

$$
\frac{1}{1+K} D_{1}(x, T x)-D_{1}(x, y) \notin \operatorname{int} P \quad \Longrightarrow \quad D_{1}(T x, T y) \ll \frac{1}{K} D_{1}(x, y) .
$$

Then $T$ has a unique fixed point in $X$.

Note that for $K=1$ the above theorem reduces to [5, Theorem 3.8]. 


\section{Competing interests}

The authors declare that they have no competing interests.

\section{Authors' contributions}

All authors contributed equally and significantly in writing this paper. All authors read and approved the final manuscript.

\section{Author details}

${ }^{1}$ Department of Mathematics, King Abdulaziz University, P.O. Box 80203, Jeddah, 21589, Saudi Arabia. ${ }^{2}$ Faculty of Organizational Sciences, University of Belgrade, Jove llića 154, Beograd, 11000, Serbia. ${ }^{3}$ Faculty of Mathematics, University of Belgrade, Studentski trg 16, Beograd, 11000, Serbia. ${ }^{4}$ Faculty of Mechanical Engineering, University of Belgrade, Kraljice Marije 16, Beograd, 11120, Serbia.

\section{Acknowledgements}

The first author gratefully acknowledges the support provided by the Deanship of Scientific Research (DSR), King Abdulaziz University during this research. The second, third and fourth authors are thankful to the Ministry of Science and Technological Development of Serbia.

\section{Received: 5 February 2012 Accepted: 18 July 2012 Published: 31 July 2012}

\section{References}

1. Suzuki, T: A generalized Banach contraction principle that characterizes metric completeness. Proc. Am. Math. Soc. 136, 1861-1869 (2008)

2. Kikkawa, M, Suzuki, T: Some similarity between contractions and Kannan mappings. Fixed Point Theory Appl. 2008, Article ID 649749 (2008). doi:10.1155/2008/649749

3. Popescu, O: Two fixed point theorems for generalized contractions with constants in complete metric spaces. Cent. Eur. J. Math. 7, 529-538 (2009)

4. Suzuki, T: A new type of fixed point theorem in metric spaces. Nonlinear Anal. 71, 5313-5317 (2009)

5. Đorić, D, Kadelburg, Z, Radenović, S: Edelstein-Suzuki-type fixed point results in metric and abstract metric spaces. Nonlinear Anal. 75, 1927-1932 (2012)

6. Huang, LG, Zhang, X: Cone metric spaces and fixed point theorems of contractive mappings. J. Math. Anal. Appl. 332(2), 1468-1476 (2007)

7. Zabrejko, PP: K-metric and K-normed linear spaces: survey. Collect. Math. 48, 825-859 (1997)

8. Khamsi, MA: Remarks on cone metric spaces and fixed point theorems of contractive mappings. Fixed Point Theory Appl. 2010, Article ID 315398 (2010). doi:10.1155/2010/315398

9. Khamsi, MA, Hussain, N: KKM mappings in metric type spaces. Nonlinear Anal. 73, 3123-3129 (2010)

10. Radenović, S, Kadelburg, Z: Quasi-contractions on symmetric and cone symmetric spaces. Banach J. Math. Anal. 5, 38-50 (2011)

11. Hussain, N, Shah, MH: KKM mappings in cone b-metric spaces. Comput. Math. Appl. 62, 1677-1684 (2011)

12. Jovanović, M, Kadelburg, Z, Radenović, S: Common fixed point results in metric type spaces. Fixed Point Theory Appl. 2011, Article ID 978121 (2011). doi:10.1155/2011/978121

13. Czerwik, S: Contraction mappings in b-metric spaces. Acta Math. Inform. Univ. Ostrav. 1, 5-11 (1993)

14. Cvetković, AS, Stanić, MP, Dimitrijević, S, Simić, Su: Common fixed point theorems for four mappings on cone metric type space. Fixed Point Theory Appl. 2011, Article ID 589725 (2011). doi:10.1155/2011/589725

15. Shah, MH, Simić, S, Hussain, N, Sretenović, A, Radenović, S: Common fixed point theorems for occasionally weakly compatible pairs on cone metric type spaces. J. Comput. Anal. Appl. 14, 290-297 (2012)

16. Jeong, GS, Rhoades, BE: Maps for which $F(T)=F\left(T^{n}\right)$. Fixed Point Theory Appl. 6, 87-131 (2005)

17. Farajzadeh, AP, Amini-Harandi, A, Baleanu, D: Fixed point theory for generalized contractions in cone metric spaces. Commun. Nonlinear Sci. Numer. Simul. 17, 708-712 (2012)

doi:10.1186/1687-1812-2012-126

Cite this article as: Hussain et al.: Suzuki-type fixed point results in metric type spaces. Fixed Point Theory and Applications 2012 2012:126.

\section{Submit your manuscript to a SpringerOpen ${ }^{0}$ journal and benefit from:}

- Convenient online submission

- Rigorous peer review

- Immediate publication on acceptance

- Open access: articles freely available online

- High visibility within the field

- Retaining the copyright to your article

Submit your next manuscript at $>$ springeropen.com 